\title{
Variability of the EUV quiet Sun emission and reference spectrum using SUMER
}

\author{
M. Kretzschmar ${ }^{1, \star}$, J. Lilensten ${ }^{1}$, and J. Aboudarham ${ }^{2}$ \\ ${ }^{1}$ Laboratoire de Planétologie de Grenoble, Bât. D de Physique, BP 53, 38041 Saint-Martin-d'Hères Cedex, France \\ ${ }^{2}$ LESIA, Observatoire de Paris, 92190 Meudon, France
}

Received 28 April 2003 / Accepted 27 January 2004

\begin{abstract}
This paper aims to build a quiet Sun EUV reference spectrum from the SOHO - SUMER database. We make use of 750 quiet Sun spectra spread over a large range of solar activity. They are carefully calibrated both in terms of frequency and intensity. The data do not show any noticeable variations versus the solar activity. This allows us to build a quiet Sun reference spectrum from data scattered over any solar activity level. We consider separately the continuum, the thick-line spectra, and the thin-line spectra. The former is computed from an average of the H Lyman and C I continua. The thick lines are directly assembled from the SUMER files. To retrieve a broad set of thin lines, we use the SUMER measurements to compute a mean Differential Emission Measure, which is then inverted. This results in a reference spectrum for the Quiet Sun, which is compared to previous studies.
\end{abstract}

Key words. Sun: UV radiation

\section{Introduction}

The extreme ultraviolet (EUV) solar flux is energetic enough to ionise the planetary upper atmospheres. It constitutes the major source of the diurnal ionospheres. The rapid growth of space weather makes it necessary to determine precisely and in absolute values.

Because the solar EUV flux is absorbed by the upper terrestrial atmosphere, it can only be observed from space. This reinforces the need for models. Most of the current models rely on a few experiments taken onboard the Atmosphere Explorer (AE) missions (i.e. Hinteregger et al. 1981). A first reference flux SC\#21REF was assembled from measurements performed in July $1976\left(f_{10.7}=70\right)$ (Hinteregger 1976). An extrapolation model - SERF1 (Hinteregger 1981) - allows estimation of the flux during other periods of solar activity. Torr et al. (1979) and Torr \& Torr (1985) proposed two EUV reference fluxes for aeronomy called F79050N $\left(f_{10.7}=243\right)$ and SC\#REFW $\left(f_{10.7}=68\right)$. The EUV spectrum was split in 37 bins including wavelength intervals as well as spectral lines. The authors suggested deducing fluxes at other activity levels with an exponential interpolation between the two reference fluxes. This work showed itself to be extremely useful. One of its qualities was that the authors proposed the corresponding absorption and ionization cross sections for the major thermospheric species.

Send offprint requests to: M. Kretzschmar, e-mail: mkretschmar@solar. standford.edu

* Present address: IFSI/CNR, via del Fosso del Cavaliere 100, 00133 Roma, Italy.
Tobiska (1991) and Tobiska \& Eparvier (1998) developed a model called EUV97, which takes data from other sources into account: SME, OSO; AEROS; rockets and ground-based facilities. This model takes into account the solar emission zone (i.e. chromosphere, transition region or corona) of numerous lines. It proposes a formula to retrieve the solar flux given the decimetric index and its average. A new version, SOLAR2000, has recently been developed. It uses a new input parameter named E10.7, computed from a previous version of the code (Tobiska et al. 2000). The second improved model is EUVAC (Richards et al. 1994). Its main difference from previous models is the reference flux chosen, and the interpolation formula. The coronal flux is also constrained to be at most $80 \%$ of the total.

Finally, Warren et al. (1996) have proposed a different approach to model the solar EUV flux by combining a spectral emission line database, solar emission-measure distributions, and estimates from ground-based solar images of the fraction of the Sun covered by the various types of activity to synthesize the irradiance. The goal was to determine a way to estimate the irradiance from EUV line emission formed in the upper chromosphere and lower transition region from the $\mathrm{Ca}$ II $\mathrm{K}$-line through the model. It requires building spectra for typical solar areas, such as quiet area, coronal hole, and active region. Based on this approach, the emission measure was derived from the spectrum of a portion of the quiet solar disk measured with the Harvard instrument onboard Skylab, and compilations of atomic data (Warren et al. 1998a,b). Hereafter we refer to this work as the NRLEUV quiet Sun spectrum. 
This last method looks to be very promising. It raises new questions, among which is the representativeness of the spectra for the different solar areas. The present work contributes to the effort for improving the modelling of the solar EUV flux. More specifically, it aims at defining a quiet Sun EUV reference spectrum, in the context of the solar EUV flux modelling using the differential emission measure (DEM) method for the thin lines. For this purpose, we use the recent solar data from the well calibrated spectrometer SUMER onboard SOHO. Section 2 presents the instrument and the reduction procedure. In Sect. 3, we discuss the results of this reduction procedure and the variability of the observed spectral line intensities. In Sect. 4, we use these intensities to compute a synthetic spectrum through the determination of a differential emission measure. Optically thick lines and continuum are taken into account to derive the reference spectrum. Section 5 compares our results with previous work and discusses the implications for the modelling of the solar EUV flux.

\section{Data and processing}

\subsection{The data}

The SOHO/SUMER instrument (Wilhelm et al. 1995) is a normal incidence spectrometer with two alternate detectors working at UV wavelengths, from $50 \mathrm{~nm}$ to $161 \mathrm{~nm}$. The two photon-counting detectors ("A" and "B") have 1024 spectral and 360 spatial pixels; they have two different photocathode areas and a Lyman $\alpha$ attenuator on the edge. Several slits are available, some of them with angular dimensions of $4^{\prime \prime} \times 300^{\prime \prime}$, $1^{\prime \prime} \times 300^{\prime \prime}$ and $1^{\prime \prime} \times 120^{\prime \prime}$. For all of the present data, the slits are North-South oriented. Spatial and spectral resolutions of each pixel are respectively about $1^{\prime \prime}$ and $0.044 \AA$ (in first order).

For our purpose, the use of the SUMER instrument has various advantages: (i) the calibration has been checked several times through the observation of well-known stable stars, computation of line intensity ratios, and comparison with the SOLSTICE instrument (Wilhelm et al. 1999). This led to an uncertainty of $15 \%$ and $33 \%$ for detector A before and after SOHO recovery respectively, and $20 \%$ and $36 \%$ for detector B (Wilhelm et al. 2000); (ii) SUMER data are available during the whole ascending phase of Solar cycle 23; (iii) all the SUMER data used are in the public domain and were retrieved from the MEDOC SOHO database (http: //www . medoc-ias.u-psud. fr).

For the present study, we selected about 750 SUMER files from 1996 to 2001, ranging from 750 to $1100 \AA$. Figure 1 shows their spectral coverage versus time. The choice of the upper wavelength limit is motivated by ionospheric study requirements. The lower limit corresponds to the compromise between the lower wavelength for first order observations with SUMER (660 $\AA$ with detector B) and limitations due to the amount and availability of data. We selected uniformly spread data over time in order to get an homogeneous set of observations during the selected period. Since SUMER reduced its observations on the solar disk with the increasing solar activity and lifetime of the mission, we have taken a smaller number of observation files during the first year of the SOHO mission,

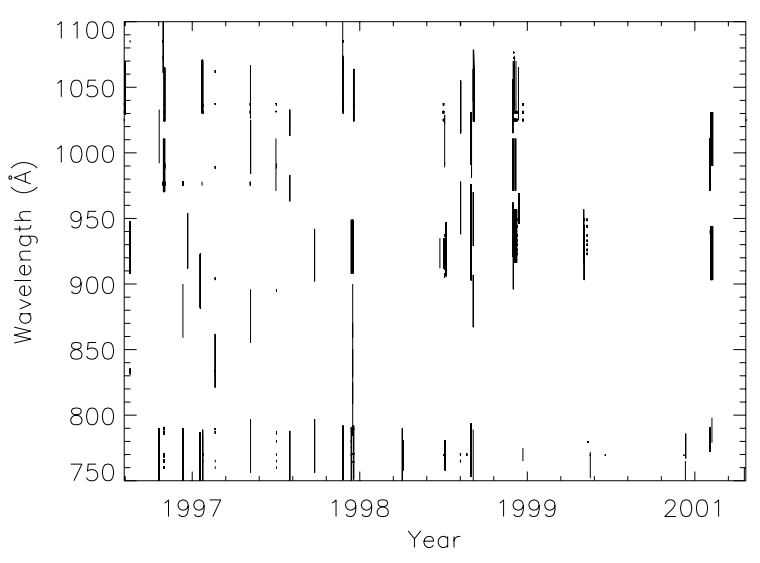

Fig. 1. Spectral coverage versus date of quiet Sun observation contained in the selected files.

with regard to what was available. This provides an equilibrium between the amount of observations at different times of the solar cycle. Their variability and therefore the variability of what is called the quiet Sun area (sometimes also shortened to "quiet Sun") is discussed in Sect. 3 and the standard deviations are shown in Tables 1 and 2.

Finally, we used EIT images (Delaboudinière et al. 1995) to identify the emission region of each file. SOHO/EIT works at 4 wavelengths (Fe IX-X at $171 \AA$, Fe XII at $195 \AA$, Fe XV at $284 \AA$ and $\mathrm{He}$ II at $304 \AA$ ) corresponding to various temperatures (respectively $1.3 \times 10^{6} \mathrm{~K}, 1.6 \times 10^{6} \mathrm{~K}, 2.0 \times 10^{6} \mathrm{~K}$ and $8.0 \times 10^{4} \mathrm{~K}$ ). Thus the whole-Sun images from EIT provide the context of the observations. We classified the emission region of each SUMER spectrum into four areas: quiet Sun area, coronal hole, active region, and other. This last area contains emission regions which where identified as a mix of two or more of the three previous areas, as well as emission regions containing particular features (i.e. filament, or case where a part of the SUMER slit was above the limb). All 4 types of emission region were identified by looking directly at the nearest EIT images (see Fig. 4 for an example); with the possibility to classify a region in the "other" category when the identification is unsure, this method is suitable to distinguish between the 3 traditional categories of emission region. Since the SUMER spectrometer has very sensitive detectors, only a few observations of active regions are available. Moreover, there are very few hot coronal lines in the spectral range used; these lines are very useful to characterize coronal holes, since their intensities are lower in coronal holes than in quiet areas. For these reasons, we only deal here with quiet Sun observations, which leaves us with about 400 SUMER files.

\subsection{Processing}

The first step when dealing with the SUMER data is to correct the count rate. This is done using IDL routines written by the SUMER team. The response of the detector is nonuniform at scales of about 20 pixels or less. This effect is corrected for each file using the closest flat-field matrix acquired by SUMER. The "Dead time" effect on the detector response, which happens when the total count rate exceeds 
Table 1. The first two columns are explicit. The third column shows the number of SUMER data files used to compute the average intensity. The fourth column shows (in percent) the statistical standard deviation of the averaged intensity, while the fifth column shows the averaged intensity. The sixth column shows intensity values from NRLEUV. The seventh column gives, when available, values from: ${ }^{1}$ Wilhelm et al. (1998), observed near disk center. ${ }^{2}$ Wilhelm et al. (1998), mean intensity deduced from full Sun observation. All intensities are in $\mathrm{mW} \mathrm{m} \mathrm{m}^{-2} \mathrm{sr}^{-1}$.

\begin{tabular}{|c|c|c|c|c|c|c|}
\hline \multicolumn{7}{|c|}{ Optically thick lines observed with SUMER } \\
\hline$\lambda(\AA)$ & Ion & $N$ & $\sigma(\%)$ & $I_{\mathrm{obs}}$ & $I_{\text {nrleuv }}$ & Others \\
\hline 917.18 & H I & 50 & 25 & 16.6 & 16.5 & \\
\hline 918.13 & H I & 50 & 24 & 19.5 & 17.0 & \\
\hline 919.34 & H I, O I & 50 & 64 & 15.9 & 18.4 & \\
\hline 920.96 & $\mathrm{H} \mathrm{I}$ & 50 & 24 & 21.6 & 18.9 & \\
\hline 921.57 & O I & 50 & 41 & 1.7 & & \\
\hline 922.02 & N IV, O I & 40 & 35 & 8.5 & 1.0 & \\
\hline 923.21 & H I, O I & 31 & 20 & 32.1 & 22.8 & \\
\hline 924.95 & O I & 31 & 25 & 2.8 & & \\
\hline 925.44 & O I & 31 & 28 & 1.4 & & \\
\hline 926.24 & H I, He II & 31 & 45 & 26.9 & 24.2 & \\
\hline 926.91 & O I & 31 & 25 & 2.0 & & \\
\hline 927.88 & He II & 31 & 58 & 1.7 & & \\
\hline 928.47 & $\mathrm{Fe}$ III & 31 & 33 & 0.9 & & \\
\hline 929.52 & O I, Fe III & 31 & 40 & 4.0 & & \\
\hline 930.79 & H I, He II & 31 & 20 & 38.2 & 30.5 & \\
\hline 931.45 & O I, O I & 31 & 22 & 3.1 & & \\
\hline 932.25 & O I & 31 & 30 & 1.0 & & \\
\hline 934.70 & Fe III & 31 & 44 & 0.9 & & \\
\hline 935.19 & O I & 30 & 37 & 1.6 & & \\
\hline 936.63 & O I & 30 & 27 & 4.4 & & \\
\hline 937.80 & H I & 30 & 23 & 59.3 & 48.5 & $37.7^{1}, 59.1^{2}$ \\
\hline 938.65 & O I & 30 & 32 & 2.8 & & \\
\hline 939.23 & O I & 30 & 25 & 2.4 & & \\
\hline 939.84 & O I & 30 & 26 & 1.5 & & \\
\hline 942.54 & He II & 30 & 58 & 3.6 & & \\
\hline 945.28 & C I & 17 & 36 & 1.1 & & \\
\hline 945.54 & C I & 17 & 25 & 1.2 & & \\
\hline 946.20 & C II & 17 & 23 & 1.3 & & \\
\hline 946.65 & $\mathrm{Mg}$ II & 17 & 14 & 0.7 & & \\
\hline 948.68 & O I & 15 & 17 & 4.6 & & \\
\hline 949.75 & H I & 15 & 14 & 83.3 & 82.1 & $59.1^{1}$ \\
\hline 950.73 & O I & 15 & 16 & 5.9 & & \\
\hline 952.35 & O I & 15 & 11 & 1.7 & & \\
\hline 952.94 & O I & 15 & 18 & 1.1 & & \\
\hline 953.41 & N I & 3 & 14 & 0.7 & & \\
\hline 953.66 & N I & 3 & 12 & 0.8 & & \\
\hline 953.97 & N I & 3 & 12 & 0.8 & & \\
\hline 958.67 & He II & 3 & 16 & 4.3 & & \\
\hline 963.99 & N I & 3 & 6 & 1.6 & & \\
\hline 964.63 & N I & 3 & 8 & 1.2 & & \\
\hline 968.95 & $\mathrm{Fe}$ III & 3 & 8 & 1.2 & & \\
\hline 971.72 & O I & 12 & 29 & 12.4 & & \\
\hline
\end{tabular}

Table 1. continued.

\begin{tabular}{llccccc}
\hline \hline \multicolumn{6}{c}{ Optically thick lines observed with SUMER } \\
\hline$\lambda(\AA)$ & Ion & $N$ & $\sigma(\%)$ & $I_{\text {obs }}$ & $I_{\text {nrleuv }}$ & Others \\
\hline 972.56 & H I & 12 & 21 & 208.6 & 169.0 & $136.0^{1}$ \\
973.23 & O I & 12 & 29 & 9.7 & & \\
973.89 & O I & 12 & 27 & 4.7 & & \\
977.96 & O I & 12 & 38 & 7.1 & & \\
981.37 & Fe III & 12 & 48 & 5.0 & & \\
983.86 & Fe III & 12 & 46 & 4.9 & & \\
985.82 & Fe III & 12 & 45 & 3.3 & & \\
990.19 & O I & 12 & 22 & 10.9 & & \\
990.76 & Fe III & 12 & 27 & 6.5 & & \\
992.36 & He II & 13 & 45 & 10.1 & & \\
992.68 & Si II & 13 & 29 & 4.7 & & \\
993.09 & Fe III & 13 & 43 & 3.2 & & \\
997.09 & Fe III & 13 & 47 & 3.6 & & \\
999.50 & O I & 13 & 26 & 8.6 & & \\
1000.16 & Ar VI & 13 & 36 & 3.0 & & \\
1017.28 & Fe III & 4 & 36 & 1.1 & & \\
1017.74 & Fe III & 3 & 28 & 0.7 & & \\
1018.28 & Fe III & 4 & 16 & 0.6 & & \\
1025.71 & H I & 5 & 17 & 751.7 & 747.0 & $766.0^{1}$ \\
1027.41 & O I & 13 & 12 & 27.0 & & \\
1028.16 & O I & 13 & 31 & 14.1 & & \\
1039.22 & O I & 14 & 12 & 5.5 & & \\
1040.94 & O I & 16 & 15 & 5.2 & & \\
1041.69 & O I & 16 & 16 & 4.3 & & \\
1063.85 & Fe III & 16 & 25 & 6.0 & & \\
1064.61 & Fe III & 10 & 19 & 5.2 & & \\
\hline & & & & & & \\
9
\end{tabular}

$5 \times 10^{4}$ counts $^{-1}$, was corrected for, as well as drops in local detector gain. Finally, we applied a correction for a geometric distortion which makes the image of the slit shorter at the centre of the detector than close to the edges.

Once these corrections has been applied, we used the RADIOMETRY.PRO routine (with keyword "epoch 9" in most cases, see Wilhelm et al. 2000) with appropriate keywords, to convert count rates into physical units. We then have at our disposal the correct intensity for detector images contained in the files.

We end up with a very large amount of data. It is therefore necessary to develop an automatic reduction procedure.

The SUMER data contained in each file is a 3-dimensional array representing spectral and spatial (along the North-South slit) dimensions, and successive exposures. First, we average each image of the SUMER detector in time and space according to the following procedure: for each file, we add the different exposures of the detector and divide the result by the number of exposures. When the solar emission is recorded from the same zone of the Sun during the entire file, this method is equivalent to averaging the data in time. When the pointing of the SUMER instrument changes between each image (When SUMER is in RASTER mode, the east-west pointing changes 
Table 2. Calculated and observed mean intensities for optically thin lines used in the DEM computation. The first three columns are explicit. The fourth column shows the number of SUMER data files used to compute the mean intensity. The fifth column shows (in percent) the statistical standard deviation of the mean intensity. The sixth and seventh columns show the mean (or "observed") and calculated intensity. The eighth column shows their ratio.

\begin{tabular}{lllccccc}
\hline \hline Ion & $\lambda(\AA)$ & $\log (T)$ & $N$ & $\sigma(\%)$ & $I_{\text {obs }}$ & $I_{\text {calc }}$ & $I_{\text {calc }} / I_{\text {obs }}$ \\
\hline S II & 996.000 & 4.2 & 4 & 30 & 1.0 & 1.0 & 0.98 \\
C II & 1036.32 & 4.3 & 19 & 13 & 49.5 & 71.5 & 1.44 \\
N II & 1085.68 & 4.4 & 6 & 5 & 55.2 & 46.7 & 0.85 \\
S III & 1012.53 & 4.7 & 2 & 28 & 0.7 & 1.0 & 1.49 \\
N III & 764.360 & 4.9 & 55 & 24 & 5.3 & 5.7 & 1.07 \\
N III & 763.340 & 4.9 & 53 & 25 & 2.8 & 2.9 & 1.05 \\
S IV & 1072.98 & 5.0 & 13 & 35 & 6.7 & 5.5 & 0.82 \\
O IV & 790.220 & 5.2 & 27 & 19 & 88.6 & 99.4 & 1.12 \\
O IV & 787.700 & 5.2 & 31 & 30 & 46.5 & 55.0 & 1.18 \\
S V & 786.480 & 5.2 & 43 & 29 & 27.5 & 16.2 & 0.59 \\
N IV & 765.150 & 5.2 & 68 & 23 & 63.3 & 45.8 & 0.72 \\
O V & 759.440 & 5.4 & 53 & 27 & 4.0 & 4.3 & 1.07 \\
O V & 760.450 & 5.4 & 65 & 24 & 17.8 & 20.0 & 1.13 \\
O V & 762.000 & 5.4 & 53 & 26 & 5.2 & 5.3 & 1.02 \\
O V & 758.680 & 5.4 & 53 & 24 & 5.3 & 5.7 & 1.07 \\
O VI & 1037.60 & 5.5 & 28 & 24 & 150.0 & 93.1 & 0.62 \\
O VI & 1031.93 & 5.5 & 28 & 18 & 337.4 & 186.8 & 0.55 \\
Ne VIII & 780.330 & 5.8 & 53 & 31 & 29.1 & 33.0 & 1.13 \\
Ne VIII & 770.410 & 5.8 & 68 & 30 & 61.4 & 65.8 & 1.07 \\
Mg VIII & 772.260 & 5.9 & 9 & 50 & 1.4 & 2.3 & 1.64 \\
S X & 776.370 & 6.1 & 52 & 33 & 0.9 & 0.8 & 0.85 \\
Si XII & 499.410 & 6.3 & 1 & 30 & 51.3 & 51.1 & 1.00 \\
\hline
\end{tabular}

${ }^{1}$ Intensity for Si XII line comes from Vernazza \& Reeves (1978). We assume $\sigma=30 \%$ for this line. All intensities are in $\mathrm{mW} \mathrm{m}^{-2} \mathrm{sr}^{-1}$.

by a few arcseconds between each exposure), this averages the data both in time and space. Finally, we also average the intensity spectra over the whole observed zone, either 120 or $360^{\prime \prime}$ depending on the slit used by SUMER for the observation. We are then left with one unidimensional array of spectral intensity. In this way, we trade in space and time resolution for noise reduction and homogenization; this is not restrictive since we are mainly interrested in building an averaged quiet Sun intensity and studying its variation over different solar activities (i.e. over a time scale of the order of the solar cycle). However, the relevance of this averaging process and the representativeness of the averaged intensities will be discussed in Sect. 3, by looking at the intensity distribution.

To identify and fit the spectral lines, we first developed the following procedure to achieve the wavelength calibration of each file:

(i) we made a manual calibration of eight files covering the whole wavelength range ( $750 \AA-1100 \AA$ ). These calibrated files serve as spectral reference files for the remainder of the processing;

(ii) during the automatic procedure, we computed the intercorrelation function between the new file and the reference files. This gave the correct wavelength scale of the new file.
The correlation coefficient associated with this wavelength calibration is larger than 0.9 , except of course above the limb where new lines appear. So we only retained the spectra recorded on the solar disk. The line identification is based on Kelly (1987), Feldman et al. (1997) and Curdt et al. (1997). Recently, Curdt et al. (2001) published an up to date line list from SUMER data. Our identification is in total agreement with their work and is therefore not shown here.

The next step was to retrieve the line intensities. This was done with an automatic fitting procedure for the optically thin lines, as described below:

(i) Selection of the lines to be processed in the procedure. The lines were chosen for their ability to be fitted; an important criterium is a high ratio of the maximum value of the line to the value of the continuum, but more constraints arise from the automatic procedure used; for example, the choice of a fixed contiunum - see below - imposes the choice of lines for which such a continuum is easily defined. The values of the central wavelength and the spectral interval to be fitted for each line are deduced from the spectral reference files, since those are used for the spectral calibration. We previously refined these values by a "trial and error" method.

(ii) Line fitting during the automatic processing. The spectral calibration allows us to determine the central position of the 
line profile (assuming a Gaussian shape). The lines are then fitted over the interval deduced from the spectral reference files, transferred to the observed central position. The fits are achieved with a constant continuum plus a Gaussian. In the fit, the value of the continuum is fixed to the observed minimum intensity value in the neighbourhoud of the lines; this choice, although unusual, is a result of the observation that it improves the number of good fits in our procedure. In fact, we observed that allowing the continuum to be fitted (by a constant or quadratic form) with the Gaussian in our automatic procedure resulted in more bad results, including "exotic" continua; this can be explained by the combined effect of the number of parameters to be guessed by the fitting routine and the fixed interval to be fitted. The error introduced by this choice is small; from case studies, values of the continuum (and so of the intensity) guessed using this method and by a fitting procedure - when it works are very similar.

After the automatic processing, we carefully examined all the output parameters and curves. A few errors were found and excluded. Estimated errors in the fit are at most $15 \%$, and less than $5 \%$ in most of the cases. Again, errors are kept to a minimum because we chose the lines in order to fit the automatic processing.

Direct integration is performed for optically thick lines. This case is discussed further in Sect. 3.2.

\section{Data analysis}

In the previous section, we presented the processing of the SUMER data, going from the detector count rates to the lines intensity. It was noticed that all detector exposures in a data file were simply averaged before determining line intensities. Moreover, depending on the original purpose of the observations, the characteristics of the data acquisition by SUMER, such as the spectral range, the slit size, the exposure time, the pointing, ... are different from file to file. Then the intensities (averaged over one file) are built from different averages over time, and to a lesser extent over space in the few cases where the SUMER pointing changes between successive detector exposures. To check the effect of these differences and to investigate how the intensity set is representative of a quiet Sun area, we look at the intensity distribution. This can be done by taking for each file the measured intensity in each portion of $5^{\prime \prime}$ of the SUMER slit, which allows enough noise reduction for the fitting procedure, and enough space resolution to distinguish the network structure. For a data file corresponding to an observation with a $120^{\prime \prime}$ slit this leads to 24 intensities. Intensity histograms are shown for the N IV line at $765 \AA$ and for the $\mathrm{H}$ I line at $918 \AA$ in Fig. 2. It may be seen that these histograms are consistent with others deduced from quiet sun data (Reeves 1976), including SUMER measurements (Pauluhn et al. 2000). The lognormal shape of the histograms is a good indicator of their coherency (Pauluhn et al. 2000). The clear presence of the network structure ensures that the set of intensities is representative for the quiet Sun.
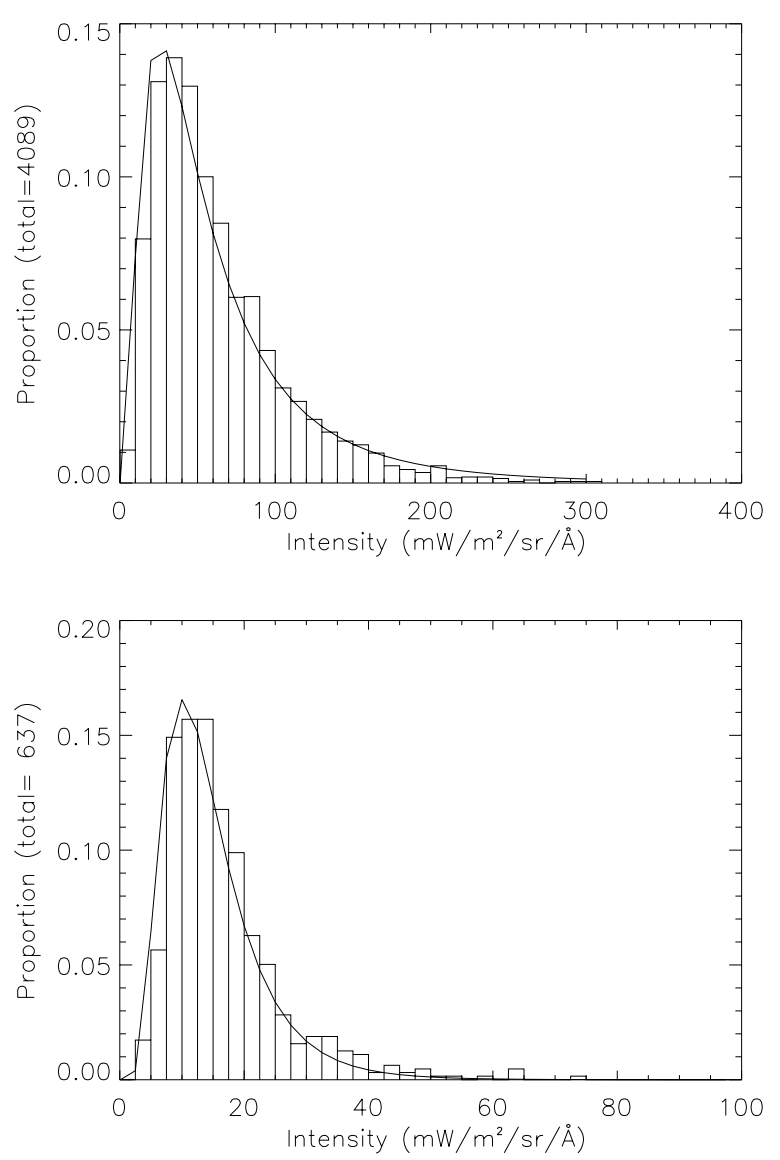

Fig. 2. Intensity histogram for (top) N IV line at $765 \AA$ and (bottom) $\mathrm{H} I$ line at $918 \AA$. The total number indicates the number of intensities in the $5^{\prime \prime}$ bands. The full line indicates the best fit with a lognormal distribution.

For some lines, the number of SUMER data files is not big enough to recognize the usual intensity distribution. However, we have kept these lines when they were useful for the DEM computation - see Sect. 4.3 and Table 2 - or when we found no published value - see Table 1 and file numbers less than about 8 files. In the following, we work with the intensities averaged over the total observed zone in each SUMER file (also referred to one observation), either 120 or $300^{\prime \prime}$ depending on the slit used by SUMER for the observations. This allows us to study the variability of the quiet sun intensity versus the ascending phase of the solar cycle. Note that averaging these intensities is equivalent to averaging all the intensities computed in the $5^{\prime \prime}$ bins.

\subsection{Results for optically thin lines}

Figure 3 shows all the quiet Sun intensities of the N IV line at $765.15 \AA$ A. 68 measures (corresponding to 68 data files) are displayed. The intensities are plotted versus the heliocentric angle; the corresponding solar activity is represented by different symbols corresponding to various decimetric index values. Smaller symbols show the intensities transposed to the center of the solar disk by using a $1 / \cos (\theta)$ theoretical center to limb variation. 


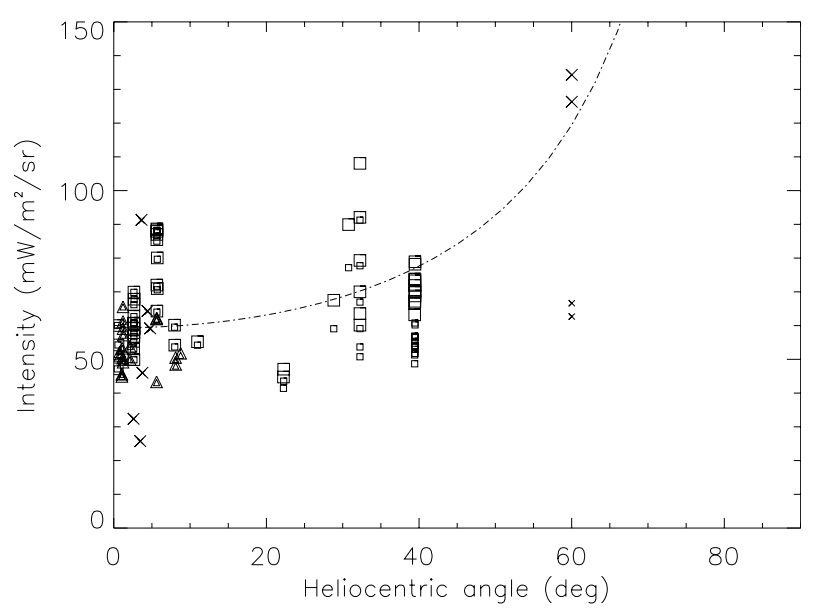

Fig. 3. Quiet area intensities for N IV (765.15 Å). Squares: quiet solar activity $\left(f_{10.7} \leq 100\right)$. Triangles: medium activity $\left(100<f_{10.7} \leq 150\right)$. Crosses: high activity $\left(f_{10.7}>150\right)$. The line shows the theoretical center-to-limb variation, while small symbols show the intensities transposed to the center of the solar disk.

This figure shows two important features: firstly, the data do not make it possible to distinguish between intensity variations due to center to limb variation and quiet Sun intensity variations due to solar activity. Next, the data show a non-negligible variation for intensities emitted in a quiet area, even at similar solar conditions and for the area close to the disk center. This variation, which can be large, is due both to intrinsic variations in and between quiet regions and to differences in the observation properties; for example, two spectra recorded from a short exposure in a small area may have very different amplitudes depending on the proportion of enhanced network present in the region or on the existence of a short-time explosive event such as blinkers; on the other hand, a spectrum recorded with a long exposure in a big solar area should be an average of all the components of the quiet Sun. The intrinsic temporal variability of the EUV quiet Sun emission has been notably studied by Brkovic̀ et al. (2002) from SUMER and CDS measurements. They found a relative intensity variation of the order of $20 \%$ for several chromospheric lines. The brightness change remains the same for time scales less and longer than 5 min. For lines emitted in the transition region, the relative variation of the intensity is of the order of $40 \%$, with a greater brightness change for time scales longer than $5 \mathrm{~min}$ and less than $80 \mathrm{~min}$. They also noted that the SUMER data have a larger variation than those from CDS. This could be explained by their different spatial resolutions.

These 3 parameters (intrinsic variation between quiet areas, center to limb variation, and variation of quiet area emission with solar activity) are very important for the reconstruction of the whole Sun emission from measurements made in small portions of the solar disk.

To study the variability of the quiet Sun intensity vs. solar activity, we split the data into 3 classes, depending on the global solar activity. We chose to use the $f_{10.7}$ index as the indicator of this activity because we aim to investigate the variation of the intensities emitted by quiet area of the Sun versus the solar cycle; the underlying question is "Do the quiet Sun

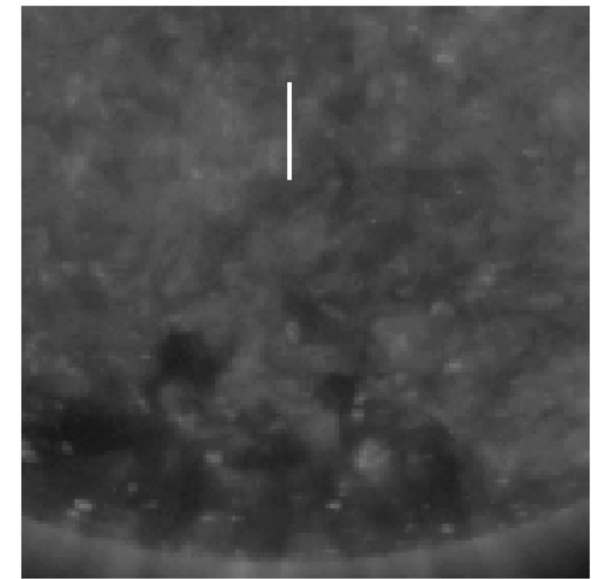

Fig. 4. Slit position for the SUMER observations on 1997 January 23, using an EIT image at $171 \AA$ (north is up). No significant changes of the appearance of the solar disk appearance were observed for the different files at this date. Note that the south edge of the Sun is at the bottom of the figure. The dark areas correspond to coronal holes.

areas take part in the increase of the solar flux with the solar cycle?". As classically made in many aeronomical studies, we have chosen 3 activity classes: quiet $\left(f_{10.7}<100\right)$, mean $\left(100 \leq f_{10.7}<150\right)$, active $\left(f_{10.7} \geq 150\right)$. The results are shown in Fig. 3. For example, the data obtained for a heliocentric angle of about $32^{\circ}$ at low activity came from measurements made on January 23, 1997, between $19 \mathrm{~h} 41 \mathrm{~m} 11 \mathrm{~s}$ and $23 \mathrm{~h} 31 \mathrm{~m} 11 \mathrm{~s}$. For the six squares, the pointing was the same; the slit position on the solar disk is shown in Fig. 4, and the emitting region is clearly a "quiet" region. Looking at several EIT images covering the whole observation time of the 6 files, we could not see any significant changes in this region. The measured intensity for the N IV $765.15 \AA$ line increases from $60.1 \mathrm{~mW} \mathrm{~m}^{-2} \mathrm{sr}^{-1}$ at $19 \mathrm{~h} 41$ to 108.1 at $22 \mathrm{~h} 14$ and then decreases to 79.3 at $23 \mathrm{~h} 31$. We carefully investigated these measurements for errors occurring in the calculation of the intensity (data corrections, error during the identification or the fitting procedure) or in the identification of the emitting region. Looking at the variation of the emission along the slit, it appears that the strong intensity for the data taken at $22 \mathrm{~h} 14$ comes from a strong emission enhancement of a small zone of about $20^{\prime \prime}$. An explosive event, or more generally the "quiet Sun activity", could be responsible for this strong increase.

The standard deviation for the intensities shown in Fig. 3 (not corrected from the center-to-limb variation) is $28 \%$, while the mean relative deviation is about $20 \%$. These variations confirm the importance of averaging intensities to retrieve typical values. Moreover, the small explosive events surrounded by a large quiet area, such as the one discussed above, could not be detected as an "active region component" from ground-based solar measurements to determine the area proportion of the solar disk occupied by the different activity features (i.e. quiet Sun, active region, and coronal hole). Therefore, we include them in our set of "quiet Sun" intensities.

As noted above, intensity variations due to center-to-limb effect and solar cycle can be difficult to distinguish in our dataset, since unfortunately most of our measurements of quiet 
area intensities at high solar activity are far from the disk center (i.e. $\theta>50^{\circ}$ ).

It remains unclear whether quiet areas of the solar disk are involved in the whole Sun EUV intensity increase during a solar cycle. Because there was no continuous EUV measurement during the ascending phase of the solar cycle until SOHO, we may refer to studies of the chromospheric network variability. White \& Livingston (1981) have studied Ca II H and K profiles from minimum to maximum of solar cycle 21 and did not observe any change in a $1^{\prime} \times 3^{\prime}$ quiet region at disk center, suggesting no long term variation for the quiet network. This result was confirmed by Labonte \& Howard (1982) using fulldisk Mount Wilson daily magnetograms. On the other hand, Kariyappa \& Sivaraman (1994) found that the network area was anti-correlated with the solar cycle, from Ca II spectroheliograms covering the period 1957-1983. Finally, Pauluhn \& Solanki (2002) have studied quiet Sun EUV intensity changes from 1996 to 2000 with SUMER and CDS data. They found a possible increase of quiet Sun He I $584 \AA$ intensity with the solar cycle, but this result is subject to a large measurement uncertainty. The amount of the increase is estimated to be $+17 \% \pm 10 \%$ with CDS and $+22 \% \pm 25 \%$ with the SUMER measurements. Note that when we apply a center to limb correction to intensity values, it appears in most cases that high intensities recorded at high activity have similar values as intensities measured near the disk center. This case is illustrated by the two measurements in Fig. 3 at $\theta \simeq 60^{\circ}$. However, since it is unclear if we must keep high intensities recorded at large $\theta$ to compute significant averaged quiet Sun intensities, we only keep those at $\theta \leq 40^{\circ}$; this limits the theoretical variation due to the center to limb effect to about $\frac{1}{\cos \left(\theta=40^{\circ}\right)} \simeq 1.3$. Since this is of the same order as the dispersion of intensities (Fig. 3), we do not apply the correction.

We may now look at the intensities recorded at low and high solar activity, at similar $\theta$. The example given in Fig. 3 is characteristic of other lines, and it was not possible to detect any increase of the quiet Sun intensity with solar activity. If this increase is present, as suggested by Pauluhn \& Solanki (2002), it is hidden by the uncertainty and the dispersion of the data.

Intensities averaged over the whole data set are shown in Table 2 with their standard deviations, for all optically thin lines. One may check that the case of the N IV $765.15 \AA$ line is typical.

\subsection{Results for optically thick lines}

For optically thick lines, the intensity is computed through direct integration, using the following procedure: once the spectrum containing the optically thick line to process is calibrated using the reference spectrum, we know the position of the center of the line with enough precision. We then compute its intensity by integrating the data over a fixed spectral range, determined from the spectral reference files. Since the spectral calibration may differ a little between different files, this method may introduce additional variability in the intensities of the lines. We check this by observing all the integrated

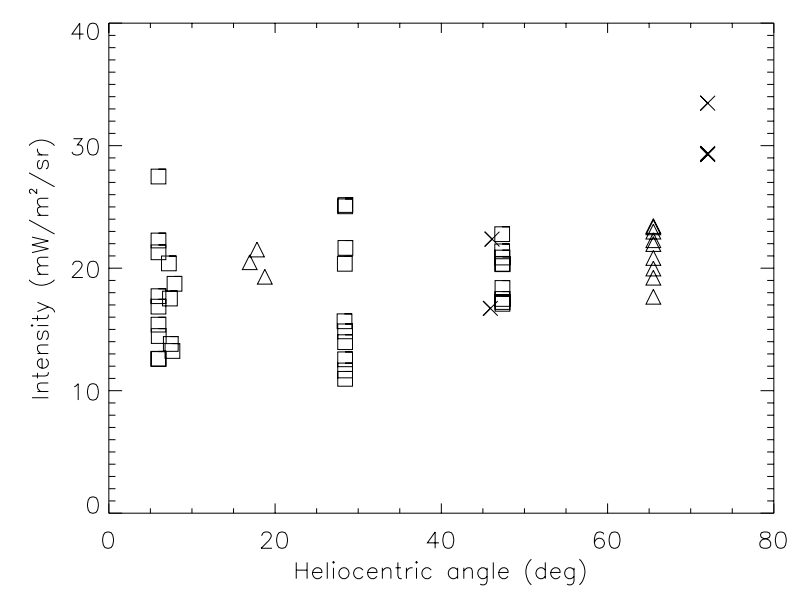

Fig. 5. Quiet area intensities for H I (918.13 $\AA$ ). Squares: quiet solar activity $\left(f_{10.7} \leq 100\right)$. Triangles: medium activity $\left(100<f_{10.7} \leq 150\right)$. Crosses: high activity $\left(f_{10.7}>150\right)$.

profiles in the case of the H I $938 \AA$ line; the method introduces negligible errors.

In Fig. 5, all the intensity values for the H I $918.13 \AA$ line are plotted versus the heliocentric angle at the center of their emission zone. As in Fig. 3, we cannot see any tendency of the quiet Sun intensity to vary as a function of solar activity or heliocentric angle. This is representative of the other optically thick lines in our data set.

As in the case of the optically thin lines, we must take into account the center to limb variation to compute the average intensity. Wilhelm et al. (1998) found little or no limb brightening for lines emitted by Si I, He I, and H I ions, compared to the case of optically thin lines. Warren et al. (1998c) found a brightening of approximately $20 \%$ near the limb for the H Lyman lines; this increase is of the order of the intensity variation on the solar disc. No brightening was found by Warren et al. for the C II $1036 \AA$ line and the Lyman continuum. SUMER data used in our study are consistent with these results (as shown in Fig. 5 in the case of H I $918 \AA$ ). Therefore, we assume that there is no significant center to limb variation for optically thick lines and continua (including the C I continuum), and we compute the mean intensity from all the measured values.

\section{Quiet Sun reference spectrum}

In the previous section we did not find any significant variation of the quiet Sun flux vs. solar activity. This allows us to build a reference quiet Sun spectrum from data taken at different solar activity periods. The solar EUV spectrum may be decomposed into three main features: optically thin emission lines, optically thick emission lines, and continuum.

\subsection{Continuum}

We want know to deduce a mean value of the $\mathrm{H}$ Lyman and C I continua. The spectral calibration is accurate enough to allow a computation in $1 \AA$ width bins. Assuming no center to limb variation for continua (see the end of Sect. 3.2), 

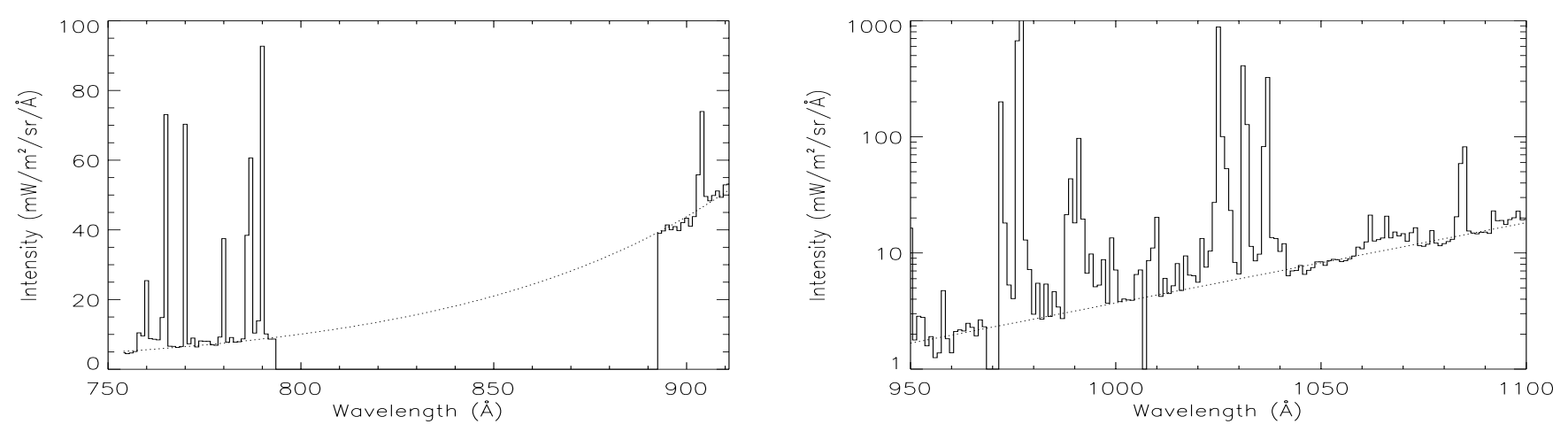

Fig. 6. Fit of the continuum background. The full line is the mean SUMER spectrum at $1 \AA$ resolution. The dotted line represents the fitted continuum. In the region between 793 and $892 \AA$, there is a lack of data corresponding to our selection criteria.

we average 188 records of the solar disk without restriction on the heliocentric angle value. The continua are then fitted from the wavelength bins where strong lines are absent, using the usual formula (i.e. Warren et al. 1998a): $I(\lambda)=I_{\mathrm{h}} \cdot \exp \left(\frac{\lambda-\lambda_{\mathrm{h}}}{\lambda_{0}}\right)$. The results are shown in Fig. 6, and values for $\lambda_{\mathrm{h}}, I_{\mathrm{h}}$ and $\lambda_{0}$ are respectively $911.75,51.9$ and 67.7 for $\mathrm{H} \mathrm{I}$, and $1101.24,17.85$ and 63.4 for $\mathrm{C} \mathrm{I}$, with the same units as previously. Only three spectra cover the range 1060-1100 А; however, the fits of the continuum with or without these spectra give the same results.

The fitted continua are slightly different from those obtained in the NRLEUV case from SKYLAB data. Both our peak and slope of the hydrogen Lyman continuum are lower. The values of the peak of the $C$ I continuum are similar both in the NRLEUV spectrum and in this work, while we find a lower slope.

\subsection{Thick lines}

Table 1 shows the average intensities of the optically thick lines deduced from SUMER measurements. When available, we give the NRLEUV values (Warren et al. 1998a,c) as well as those of Wilhelm et al. (1998); the latter were also obtained from SUMER measurements. Note that values from Wilhelm et al. (1998) observed in a region near the disk center are systematicaly lower than our values (except for Ly $\beta$ ). However, the mean intensity deduced from a quiet full Sun observation for H I $937.80 \AA$ is in agreement with our value. We believe that this is another indication of the high variability of the emission in quiet areas. In general, standard deviations of the averaged intensities obtained in this work are of the order of $25 \%$. Higher values mainly correspond to low-intensity lines (such as the Fe III $981.37 \AA$ line) or to blended lines, such as the H I and O I lines around 919.3 $\AA$. Finally, the agreement with the NRLEUV (1998c) average quiet Sun model for Lyman lines is very good. This model is based on a continuous observation sequence lasting 6h48m, resulting in 693 exposures. This sequence was observed in May, 15-16, 1996 during quiet solar conditions.

\subsection{Thin lines}

The intensities of each optically thin line may be deduced from a small number of line measurements through the computation of a Differential Emission Measure (DEM). This well-known process has been described in detail by various authors (see for example Pottasch 1963; Landi \& Landini 1997; Warren et al. 1998a). Typically, the intensity $I(\lambda)$ of an optically thin line may be written as

$I(\lambda)=\int G(\lambda, T) \xi(T) \mathrm{d} T$

where $G(\lambda, T)$ is the contribution function and depends only on the temperature and the atomic parameters of the transition, and $\xi(T)$ is the DEM. In this work, the contribution functions were computed with the help of the CHIANTI database (Dere et al. 1997). The inversion of Eq. (1) for a set of intensities leads to the DEM; in reverse, it is possible to compute the intensities of all optically thin lines from the DEM. One of the main difficulties in the computation of a correct DEM is the choice of lines. The first criterion is that they must cover a broad range of temperatures in order to determine the emission measure both for the solar transition region and the corona. The lines must be optically thin so that there is no interference with important processes such as opacity or interactions with the solar radiation field. McIntosh et al. (2000) have studied the influence of several emission lines in the spectral range of the SUMER and CDS instruments; they developed a procedure to select a subset of lines suitable for the DEM computation. It was shown that rather than individual lines, one should rely on good sets of lines rather than on individual lines. However, in our case, the aim of the inversion is to be able to recompute all the thin lines. We must then keep all the lines in the inversion procedure. Table 2 shows these lines, with their statistical characteristics and computed intensities. The entire hightemperature range of the emission measure is clearly dependent on the intensity of the Si XII line, which is from Vernazza \& Reeves (1978) SKYLAB measurements, since in our intensity set there is no line that is formed at a sufficient temperature. That Si XII line is formed at $2 \times 10^{6} \mathrm{~K}$. It is Li-like and therefore somewhat suspect. However, this line is in the optimal set of 30 lines among 133 found by McIntosh et al. (2000) for the DEM computation; it was also selected in every run of their procedure as one of the lines which is suitable to the computation of the DEM. The intensity of this line is also averaged and then fits well into our data. For each line we first compute its contribution function; this is done using the abundances from 
Table 3. Numerical values for the quiet sun DEM. DEM is in $\mathrm{cm}^{-5} \mathrm{~K}^{-1}$.

\begin{tabular}{cc}
\hline \hline $\log (T)$ & $\log ($ DEM $)$ \\
\hline 4.2 & 24.6 \\
4.3 & 24.0 \\
4.4 & 23.5 \\
4.5 & 22.9 \\
4.6 & 22.4 \\
4.7 & 22.0 \\
4.8 & 21.6 \\
4.9 & 21.3 \\
5.0 & 21.0 \\
5.1 & 20.8 \\
5.2 & 20.7 \\
5.3 & 20.6 \\
5.4 & 20.6 \\
5.5 & 20.7 \\
5.6 & 20.7 \\
5.7 & 20.8 \\
5.8 & 20.9 \\
5.9 & 20.9 \\
6.0 & 21.0 \\
6.1 & 20.9 \\
6.2 & 20.8 \\
6.3 & 20.6 \\
6.4 & 20.2 \\
6.5 & 19.6 \\
6.6 & 18.9 \\
\hline &
\end{tabular}

Meyer (1985) and ionisation equilibria computed by Arnaud \& Rothenflug (1985), and the facilities offered by the CHIANTI database (Dere et al. 1997). We tested two inversion methods to compute the DEM from the contribution functions, a nonlinear least-squares fit assuming that the DEM varies as an exponential of a Chebyshev polynomial, and a method of direct inversion plus a smoothing condition (Craig \& Brown 1986). Finally, from the DEM, it is possible to re-compute the intensities of the emission lines. We obtain the best result with the non linear least-squares fit. The computed DEM is shown in Fig. 7, and compared to previous studies. The numerical values are displayed in Table 3.

There is a close agreement with the NRLEUV DEM, although they are computed from different data sets. The data used in the NRLEUV case consist of an averaged composite spectrum assembled from measurements made near solar minimum (between May 1974 and January 1975), while the intensities used in this work were averaged from a very large range of solar activity. The similarity of the results indicates that the DEMs and inferred thin lines are representative of an averaged quiet area on the solar disk. The computed intensities are shown in Table 2. There is a general good agreement between computed and observed intensities; but there are discrepancies for some lines. The discrepancies in the C II and

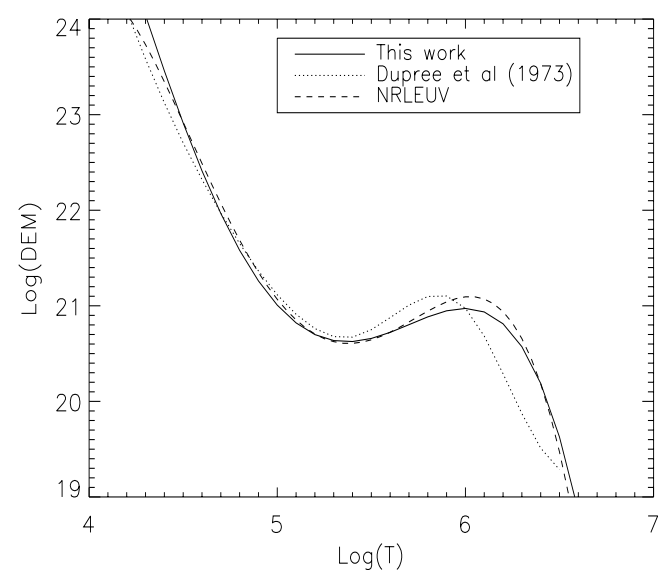

Fig. 7. Mean quiet Sun differential emission measure from SUMER.

S III lines may be explained by the possibility that these lines are not effectively thin, since they are formed at a low temperature. Disagreements for the line of the O VI ion were already noted (Judge et al. 1995). Note that the presence of these lines in the computation of the DEM does not change the shape of the DEM. Generally, the agreement is within the range found in previous studies by various authors.

\section{Comparison and discussion}

\subsection{Comparison with SUMER data}

The aim of this section is to compare our synthetic spectrum with the data from which it is deduced. This comparison allows us to check the validity of the method (i.e. DEM computation, fitted continua, and direct integration for optically thick lines). Figure 8 shows such a comparison, with a resolution of $1 \AA$. To make a more suitable comparison, we have convolved each line present in the synthetic spectrum with a gaussian profile; we have used a thermal Doppler width at ionization temperature from Arnaud \& Rothenflug (1985) and Arnaud \& Raymond (1992). One sees a very good agreement for the range 755-790 $\AA$, which is dominated by the H Lyman continuum and optically thin lines. The range $900-1100 \AA$, which is dominated by the continuum and optically thick lines, also shows a quite close agreement. Values of the computed and observed continuum are in total agreement, which is not surprising. Most of the differences are due to the inability of our reconstructed spectrum to properly take into account the correct spectral width of the optically thick lines. Thus, we have a good agreement for the $1025 \AA$ bin which contains the strong H Ly $\beta$ line, while the $1026 \AA$ bin, which contains a part of this line and a part of an $\mathrm{O}$ I line, is underestimated in our computation. C II lines around $904 \AA$ are poorly reproduced. The most probable explanation is a failure of one or more hypotheses, since this line is emitted in the high chromosphere. There is however a fairly close agreement between the two spectra, indicating that the estimate of the part of the synthetic spectrum where no data are available for the comparison should be reliable. 

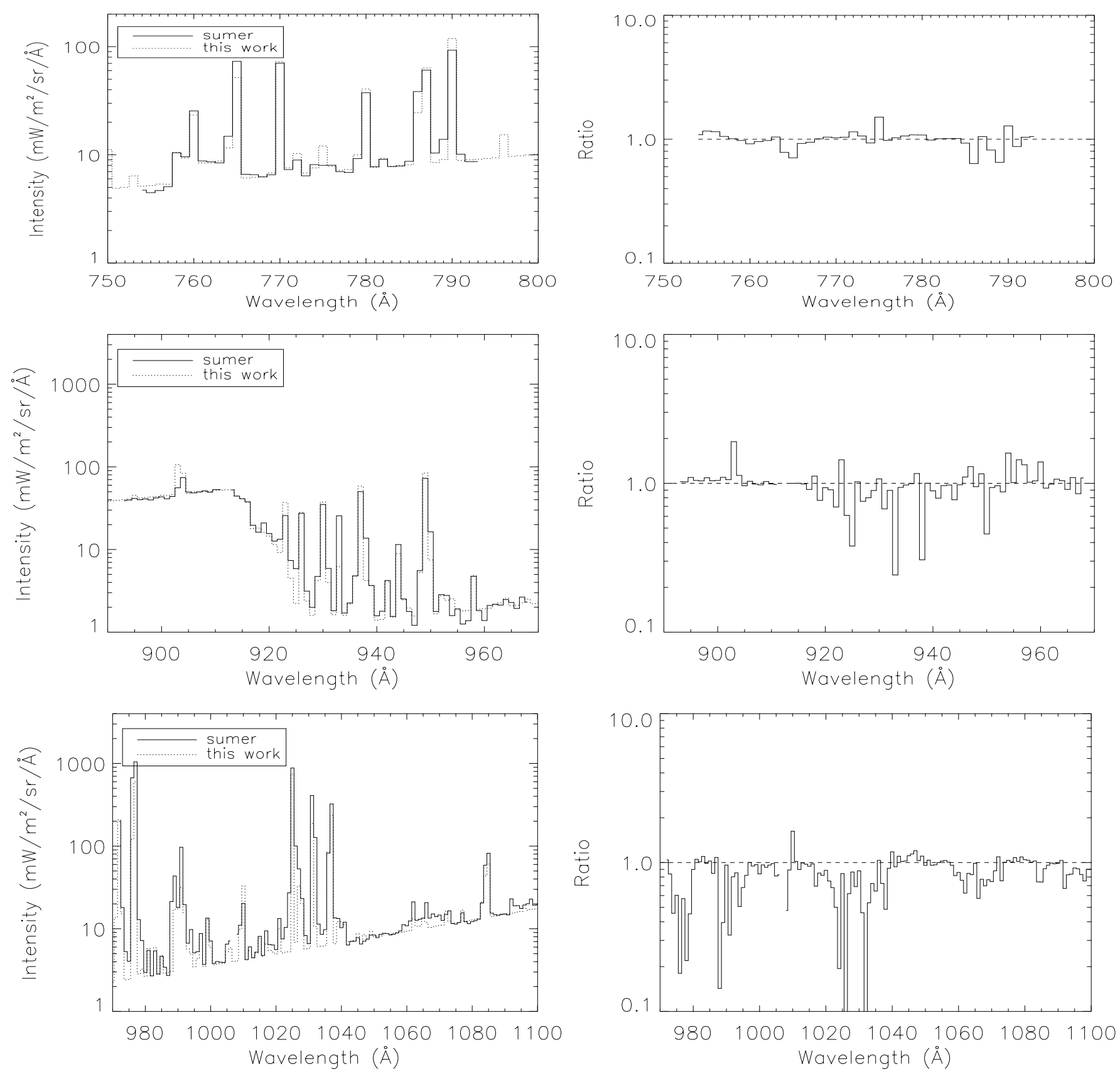

Fig. 8. Comparison of the synthetic reference spectrum with the SUMER data. Left panels show the spectrum in three different wavelength ranges; right panels show the ratio of the synthetic spectrum (labeled "this work") to the composite SUMER spectrum.

\subsection{Comparison with the NRLEUV spectrum}

For the comparison of optically thin lines, we use the lines listed in Table 7 of Warren et al. (1998a). Table 4 shows the computed intensities from our DEM, and the NRLEUV intensities for these 25 intense thin lines. The line intensities are in agreement within about $30 \%$, and up to $50 \%$ for Fe IX and Fe XIV lines. Note that NRLEUV lines were computed from atomic data included in version 1 of the CHIANTI database, and from previous work of Mewe et al. $(1985,1995)$ for the short wavelength transition of Si VII and VIII, Ni X-XIV, and Fe IX-XIV. The current work makes use of the CHIANTI version 3.02 (Dere et al. 1997; Landi et al. 1999); since the shapes of the DEM are similar (as shown in Fig. 7) and since intensities were computed with the same ionization equilibrium and solar abundances, the differences in the computed intensities are mainly due to the atomic data. Figure 9 shows the comparison of our reference spectrum with the NRLEUV spectrum in the range 500-1100 $\mathrm{A}$. We generally see a good agreement, however we may distinguish three main discrepancies. First, as discussed in Sect. 4.1, the shapes of the H I and C I continua are different. The peak of the Lyman continum is overestimated by $24 \%$ by NRLEUV, with regard to our values, while its slope is steeper. We could not find clear reasons for these disagreements. Next, several optically thin lines, which are not in the NRLEUV spectrum, appear in our computation. Namely Ca IX 

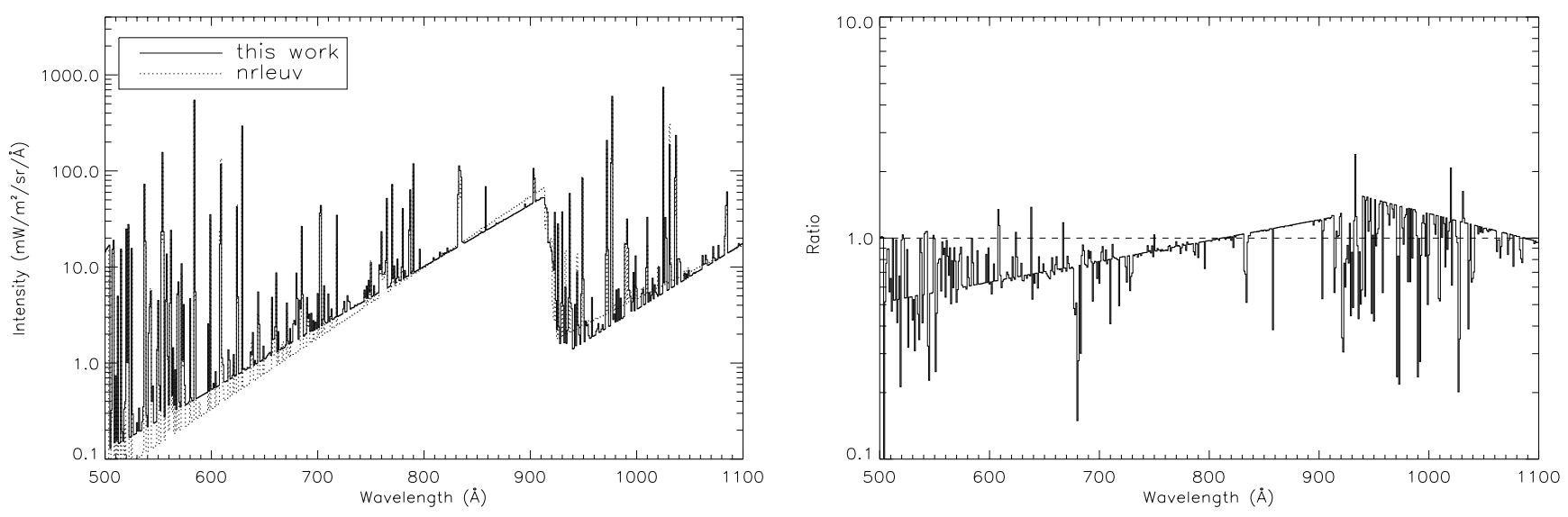

Fig. 9. Comparison of NRLEUV reference spectrum with this work. Left and right panels are defined as in Fig. 8.

Table 4. Intensity (in $\mathrm{mW} \mathrm{m}^{-2} \mathrm{sr}^{-1}$ ) for strong optically thin lines.

\begin{tabular}{|c|c|c|c|c|c|}
\hline$\lambda(\AA)$ & Ion & $\log (T)$ & $I_{\text {calc }}$ & $I_{\text {nrleuv }}$ & $r$ \\
\hline 977.02 & C III & 4.8 & 707.0 & 687.0 & 1.03 \\
\hline 171.07 & Fe IX & 5.8 & 897.3 & 586.0 & 1.53 \\
\hline 174.53 & Fe X & 6.0 & 333.9 & 436.0 & 0.77 \\
\hline 1031.9 & $\mathrm{O}$ VI & 5.5 & 186.8 & $305.0^{*}$ & 0.61 \\
\hline 177.24 & Fe X & 6.0 & 195.9 & 264.0 & 0.74 \\
\hline 180.41 & $\mathrm{Fe} \mathrm{XI}$ & 6.1 & 244.5 & 262.0 & 0.93 \\
\hline 195.12 & Fe XII & 6.1 & 329.4 & 262.0 & 1.26 \\
\hline 629.73 & $\mathrm{OV}$ & 5.4 & 293.1 & 225.0 & 1.30 \\
\hline 202.04 & Fe XIII & 6.2 & 283.0 & 208.0 & 1.36 \\
\hline 193.52 & Fe XII & 6.1 & 199.7 & 158.0 & 1.26 \\
\hline 284.16 & Fe XV & 6.3 & 257.8 & 154.0 & 1.67 \\
\hline 1037.6 & O VI & 5.5 & 93.1 & 152.0 & 0.61 \\
\hline 368.07 & Mg IX & 6.0 & 142.1 & 141.0 & 1.01 \\
\hline 1175.7 & C III & 4.8 & 138.8 & 136.0 & 1.02 \\
\hline 303.32 & Si XI & 6.2 & 128.1 & 133.0 & 0.96 \\
\hline 188.23 & $\mathrm{Fe} \mathrm{XI}$ & 6.1 & 116.5 & 124.0 & 0.94 \\
\hline 184.54 & $\mathrm{Fe} X$ & 6.0 & 73.5 & 108.0 & 0.68 \\
\hline 609.79 & $\operatorname{Mg} X$ & 6.0 & 85.4 & 90.2 & 0.95 \\
\hline 554.51 & O IV & 5.2 & 111.2 & 88.6 & 1.26 \\
\hline 790.20 & O IV & 5.2 & 99.4 & 79.6 & 1.25 \\
\hline 319.83 & Si VIII & 5.9 & 77.8 & 78.9 & 0.99 \\
\hline 211.32 & Fe XIV & 6.3 & 115.0 & 76.9 & 1.50 \\
\hline 465.22 & $\mathrm{Ne}$ VII & 5.7 & 83.9 & 74.3 & 1.13 \\
\hline 258.37 & Si X & 6.1 & 67.6 & 72.5 & 0.93 \\
\hline 315.04 & Mg VIII & 5.9 & 69.1 & 69.4 & 0.99 \\
\hline
\end{tabular}

* Intensity directly deduced from Vernazza \& Reeves (1978) in the NRLEUV spectrum. $r$ is the ratio $I_{\text {calc }} / I_{\text {nrleuv }}$.

at $530.97 \AA, \mathrm{Fe}$ XIV at $532.27 \AA$, S III for several lines near $678 \AA$, and at $710.96 \AA$, $724.29 \AA$, $725.86 \AA$, and $728.68 \AA$. Two C II lines near $858 \AA$ are also present, but since this ion more probably belongs to the chromosphere, these lines may not be optically thin and their intensities should not be adopted without caution. Lastly, the NRLEUV spectrum underestimates the line emission above $911 \AA$. This part of the spectrum is dominated by optically thick lines, such as the Lyman series and $\mathrm{H}$ or $\mathrm{O}$ lines. Optically thick lines in the NRLEUV spectrum come only from $\mathrm{H}$ and $\mathrm{He}$. This feature explains most of the lower value of the NRLEUV spectrum in some $1 \AA$ bins. This is emphasized in Fig. 10 which shows the comparison in this range of the NRLEUV spectrum with the measured SUMER spectrum in $1 \AA$ bins. The higher value in the SUMER spectrum at $939 \AA$ is due to an O I line, while the one at $942 \AA$ is due to a He II line. Other examples are the bins at $929 \AA$ with an O I line, 952 and $953 \AA$ with an O I line and three N I lines, $958 \AA$ with a He II line, or $981 \AA$ A $983 \AA$ and $985 \AA$ with three Fe III lines. Optically thick lines observed in the SUMER spectrum and included in our synthetic spectrum are listed in Table 1 with their average intensities and standard deviations.

\section{Conclusions}

In this paper, we have presented a quiet Sun reference spectrum in the EUV range. This spectrum is based on a large amount of data from the SOHO/SUMER spectrometer, spread over the ascending phase of the current solar cycle. The study of these data has not shown any evidence of an increase of the quiet Sun intensity with the solar activity. We have determined a mean differential emission measure, mean intensity for several optically thick lines, and a mean intensity for H I and C I continua for quiet areas of the solar atmosphere. We made a comparison with the NRLEUV reference spectrum for the quiet Sun. Despite the different sources of the data (i.e. SKYLAB vs. SOHO), a generally good agreement was found between the two spectra. The two DEM are very similar, but some differences were found for the optically thin emissions because of the use of more recent atomic data in this work. However, since the computation of the DEM is subject to errors (Lang et al. 1990; Craig \& Brown 1986), we think that it would be difficult to obtain a precision of more than approximately $20 \%$ for the computed intensities in the whole EUV range using this method. The continua are slightly different, and no clear explanation was found. Finally, the inclusion in our reference spectrum of several optically thick lines above $911 \AA$ and emitted by ions others than H I, improve the precision of the spectrum. 

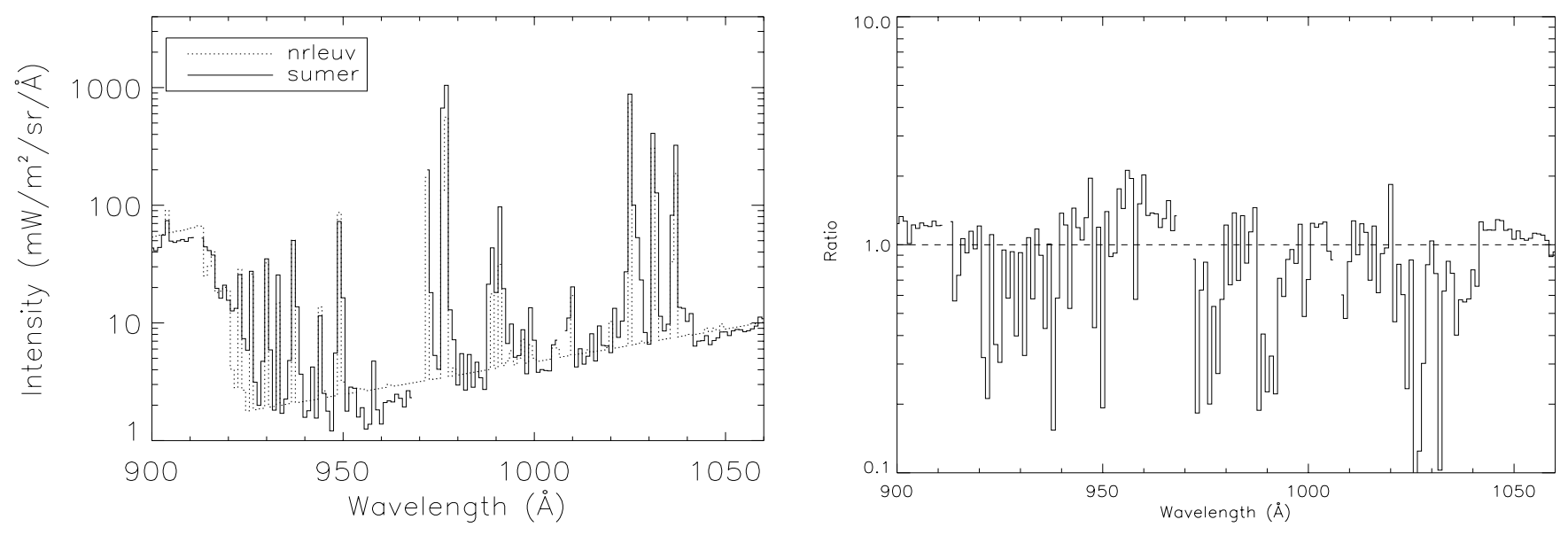

Fig. 10. Comparison of NRLEUV reference spectrum with the composite SUMER spectrum. Left and right panels are defined as in Fig. 8.

Acknowledgements. We thank P. Lantos (LESIA) for his active and strong support, and W. Kofman (LPG) for helpful discussions. We thank the MEDOC staff and the CHIANTI researchers for their help, and the staff of SUMER and EIT for providing access to their data. This work has been supported by the PNST-CNRS research program and LESIA at Paris Observatory. The computations have been performed at the Service de Calcul Intensif of the Grenoble Observatory.

\section{References}

Arnaud, M., \& Raymond, J. C. 1992, ApJ, 398, 394

Arnaud, M., \& Rothenflug, R. 1985, A\&AS, 60, 425

Brković, A., Solanki, S. K., \& Rüedi, I. 2002, A\&A, 385, 257

Craig, I. J. D., \& Brown, J. C. 1986, Inverse problems in astronomy (Bristol, UK and Boston, M.A., Adam Hilger Ltd.), 159

Curdt, W., Feldman, U., Laming, J. M., et al. 1997, A\&AS, 126, 281

Curdt, W., Brekke, P., Feldman, U., et al. 2001, A\&A, 375, 591

Delaboudinière, J. P., Artzner, G. E., Brunaud, J., et al. 1995, Sol. Phys., 162, 291

Dere, K. P., Landi, E., Mason, H. E., Monsignori Fossi, B. C., \& Young, P. R. 1997, A\&AS, 125, 149

Dupree, A. K., Huher, M. C. E., Noyes, R. W., et al. 1973, ApJ, 182, 321

Feldman, U., Behring, W. E., Curdt, W., et al. 1997, ApJS, 113, 195

Hinteregger, H. E. 1976, J. Atm. Terr. Phys., 38, 791

Hinteregger, H. E. 1981, Adv. Sp. Res., 1, 39

Hinteregger, H. E., Fukui, K., \& Gilson, B. R. 1981, Geoph. Res. Lett., 8,1147

Judge, P. G., Woods, T. M., Brekke, P., \& Rottman, G. J. 1995, ApJ, 455, L85

Kariyappa, R., \& Sivaraman, K. R. 1994, Sol. Phys., 152, 139

Kelly, R. L. 1987, Atomic and ionic spectrum lines below $2000 \AA$ A, New York, American Institute of Physics, American Chemical Society and the National Bureau of Standards

Labonte, B. J., \& Howard, R. 1982, Sol. Phys., 80, 15

Landi, E., \& Landini, M. 1997, A\&A, 327, 1230

Landi, E., Landini, M., Dere, K. P., Young, P. R., \& Mason, H. E. 1999, A\&AS, 135, 339
Lang, J., McWhirter, R. W. P., \& Mason, H. E. 1990, Sol. Phys., 129, 31

McIntosh, S. W., Charbonneau, P., \& Brown, J. C. 2000, ApJ, 529, 1115

Mewe, R., Gronenschild, E. H. B. M., \& van den Oord, G. H. J. 1985, A\&AS, 62, 197

Mewe, R., Kaastra, J. S., \& Liedahl, D. A., Update of MEKA: MEKAL, Legacy, 6, 16

Meyer, J. P. 1985, ApJS, 57, 173

Pauluhn, A., Solanki, S. K., Rüedi, I., Landi, E., \& Schühle, U. 2000, A\&A, 362, 737

Pauluhn, A., \& Solanki, S. K. 2002, ESA Publications Division, ISBN 92-9092-818-2, 227

Reeves, E. M. 1976, Sol. Phys., 46, 53

Pottasch, S. R. 1963, ApJ, 137, 945

Richards, P. G., Fennelly, J. A., \& Torr, D. G. 1994, J. Geoph. Res., 99, 8981

Tobiska, W. K. 1991, J. Atm. Terr. Phys., 53, 1005

Tobiska, W. K., \& Eparvier, F. G. 1998, Sol. Phys., 177, 147

Tobiska, W. K., Woods, T., Eparvier, F. G., et al. 2000, J. Atm. Terr. Phys., 62, 1233

Torr, M. R., \& Torr, D. G. 1985, J. Geoph. Res., 90, 6675

Torr, M. R., Torr, D. G., Ong, R. A., \& Hinteregger, H. E. 1979, Geoph. Res. Lett., 6, 771

Vernazza, J. E., \& Reeves, E. M. 1978, ApJS, 37, 485

Warren, H. P., Mariska, J. T., Lean, J., Marquette, W., \& Johannesson, A. 1996, Geoph. Res. Lett., 23, 2207

Warren, H. P., Mariska, J. T., \& Lean, J. 1998a, J. Geoph. Res., 103, 12077

Warren, H. P., Mariska, J. T., \& Lean, J. 1998b, J. Geoph. Res., 103, 12091

Warren, H. P., Mariska, J. T., \& Wilhelm, K. 1998c, ApJS, 119, 105

White, O. R., \& Livingston, W. C. 1981, ApJ, 249, 798

Wilhelm, K., Curdt, W., Marsch, E., et al. 1995, Sol. Phys., 162, 189

Wilhelm, K., Lemaire, P., Dammasch, I. E., et al. 1998, A\&A, 334 , 685

Wilhelm, K., Woods, T. N., Schühle, U., et al. 1999, A\&A, 352, 321

Wilhelm, K., Schühle, U., Curdt, W., et al. 2000, Metrologia, 37, 393 